Research Paper

Fangqin He and Lianzhong Li*

\title{
Time fractional modified KdV-type equations: Lie symmetries, exact solutions and conservation laws
}

https://doi.org/10.1515/phys-2019-0049

Received Jun 03, 2019; accepted Aug 01, 2019

Abstract: In the paper, we research a time fractional modified KdV-type equations. We give the symmetry reductions and exact solutions of the equations, and we investigate the convergence of the solutions. In addition, the conservation laws of the equations are constructed.

Keywords: Time fractional differential equations; Lie symmetry; Exact solution; Conservation laws

PACS: 02.30.Jr, 02.20.-a, 02.30.Ik, 04.20.Jb

\section{Introduction}

Nonlinear partial differential equation(NLPDE) is a kind of important mathematical model for describing the natural phenomena and mathematical physics. Over the past few years, many ordinary and partial differential equations(PDEs) were concerned by the researchers, they have obtained many good results[1 - 10]. At present, many approaches have been extensively studied for constructing exact solutions of the equation, such as the inverse scattering transformation(IST)[11], Darboux transformation method and Bäcklund transformation method[12], Hirota's bilinear method[12-14], Lie symmetry analysis[1520], CK method[21], and so on. Now, there are more and more related researches on fractional partial differential equations(FPDEs). At the same time, those methods are also widely used in solving the precise solution of these equations[6, 24, 25]. In particular, the classical Kortewegde Vries (Kdv) equations which play an important role in many mathematical and physical fields. In [19, 24 30], the authors used the power series method to solve

\footnotetext{
*Corresponding Author: Lianzhong Li: School of Science, Jiangnan University, Wuxi, 214122, China; Email: 1lz3497@163.com Fangqin He: School of Science, Jiangnan University, Wuxi, 214122, China
}

๖ Open Access. @ 2019 F. He and L. Li, published by De Gruyter. the classical Kdv equations. Meanwhile, they sought the help of the new conservation theorem which constructed the conservation laws(Cls) for the governing equations. At the same time, fractional calculus is also very popular, it has been successfully used to explain many complex nonlinear phenomena and dynamic processes in physics, engineering, electromagnetics, viscoelasticity, and electrochemistry. Inspired by the above, we considered here to study the time-fractional modified KdV-type equations, which are presented in the following form:

$$
\begin{aligned}
& \frac{\partial^{\alpha} u}{\partial t^{\alpha}}-u_{x x x}-u v u_{x}=0, \\
& \frac{\partial^{\alpha} v}{\partial t^{\alpha}}-v_{x x x}-u v v_{x}=0,
\end{aligned}
$$

in Eq. (1), $0<\alpha<1, \frac{\partial^{\alpha} \cdot}{\partial t^{\alpha}}$ is the Riemann-Liouville (RL) fractional derivatives. If $\alpha=1$, Eq. (1) becomes

$$
\begin{aligned}
& u_{t}-u_{x x x}-u v u_{x}=0, \\
& v_{t}-v_{x x x}-u v v_{x}=0
\end{aligned}
$$

Eq. 2 is a modified KdV-type equations. The modified KdV-type equations is most popular mathematical models and have been extensively investigated. And it has been applied to describe the electromagnetic waves in sizequantized films, interfacial waves in two-layer liquids, and transmission lines in the Schottky barrier. It was analyzed and studied in [24], Lie symmetry analysis, exact solutions and CLs for Eq. 2 were investigated. And Eq. (1) comes from the modified KdV-type equations by replacing its time derivative with a fractional derivative. so, we will analyze and investigate the Lie symmetries, exact solutions, CLs and the convergence of the exact solutions for Eq. (1).

In this paper, to the best of our knowledge, we apply Lie method to study Eq. (1), we get the optimal system and the exact solutions for the equation, we also give the $\mathrm{Cls}$ for the equations via a new conservation theorem.

This article is divided into the following sections: First of all, in section 2, we introduce some essential knowledge which will be used in later chapters; in section 3, we seek the help of the Lie method which can acquire the optimal system and the symmetry reductions of Eq. (1); in section 
4, on the basis of the third quarter, we calculate the exact solution of the equations; in section 5 , the convergence of the exact solutions for the equations will be investigated; in section 6, we use the symmetries and adjoint equations to construct the conservation laws, there are some conclusions and discussions in the last section.

\section{Preliminaries}

We introduce some essential knowledge about the RL fractional derivative and the Lie symmetries in the section. Firstly, the definition of the RL fractional derivative [22, 23] is as follows:

$$
D^{\alpha} f(t)= \begin{cases}\frac{d^{n} f}{d t^{n}}, & \alpha=n, \\ \frac{d}{d t^{n}} I^{n-\alpha} f(t), & 0 \leq n-1<\alpha<n,\end{cases}
$$

$n$ is a natural number and $I^{n-\alpha} f(t)$ is defined by

$$
\begin{aligned}
& I^{n-\alpha} f(t)=\frac{1}{\Gamma(n-\alpha)} \int_{0}^{t}(t-s)^{n-\alpha-1} f(s) d s, \\
& n-\alpha>0, \quad I^{n-\alpha} f(t)=f(t), \quad n-\alpha=0,
\end{aligned}
$$

where $\Gamma(n-\alpha)$ is the gamma function.

Let us consider the blow space-time FPDEs:

$$
\begin{aligned}
& D_{t}^{\alpha} u=F_{1}\left(t, x, u, v, u_{x}, v_{x}, \ldots\right),(\alpha>0) \\
& D_{t}^{\alpha} v=F_{2}\left(t, x, u, v, u_{x}, v_{x}, \ldots\right),(\alpha>0)
\end{aligned}
$$

next, we present the form of a one-parameter Lie group of infinitesimal transformations is as blow:

$$
\begin{aligned}
x^{\star} & =x+\epsilon \xi^{1}(x, t, u, v)+o\left(\epsilon^{2}\right), \\
t^{\star} & =t+\epsilon \xi^{2}(x, t, u, v)+o\left(\epsilon^{2}\right), \\
u^{\star} & =u+\epsilon \eta^{1}(x, t, u, v)+o\left(\epsilon^{2}\right), \\
r v^{\star} & =v+\epsilon \eta^{2}(x, t, u, v)+o\left(\epsilon^{2}\right), \\
\frac{\partial^{\alpha} u^{\star}}{\partial t^{\star \alpha}} & =\frac{\partial^{\alpha} u}{\partial t^{\alpha}}+\epsilon \eta^{1 \alpha, t}(x, t, u, v)+o\left(\epsilon^{2}\right), \\
\frac{\partial^{\alpha} v^{\star}}{\partial t^{\star \alpha}} & =\frac{\partial^{\alpha} v}{\partial t^{\alpha}}+\epsilon \eta^{2 \alpha, t}(x, t, u, v)+o\left(\epsilon^{2}\right), \\
\frac{\partial u^{\star}}{\partial x^{\star}} & =\frac{\partial u}{\partial x}+\epsilon \eta^{1 x}(x, t, u, v)+o\left(\epsilon^{2}\right), \\
\frac{\partial v^{\star}}{\partial x^{\star}} & =\frac{\partial v}{\partial x}+\epsilon \eta^{2 x}(x, t, u, v)+o\left(\epsilon^{2}\right), \\
\frac{\partial^{3} u^{\star}}{\partial x^{\star 3}} & =\frac{\partial^{3} u}{\partial x^{3}}+\epsilon \eta^{1 x x x}(x, t, u, v)+o\left(\epsilon^{2}\right), \\
\frac{\partial^{3} v^{\star}}{\partial x^{\star 3}} & =\frac{\partial^{3} v}{\partial x^{3}}+\epsilon \eta^{2 x x x}(x, t, u, v)+o\left(\epsilon^{2}\right),
\end{aligned}
$$

where

$$
\eta^{1 x}=D_{x}\left(\eta^{1}\right)-u_{x} D_{x}\left(\xi^{1}\right)-u_{t} D_{t}\left(\xi^{2}\right)
$$

$$
\begin{aligned}
\eta^{2 x} & =D_{x}\left(\eta^{2}\right)-v_{x} D_{x}\left(\xi^{1}\right)-v_{t} D_{t}\left(\xi^{2}\right), \\
\eta^{1 x x} & =D_{x}\left(\eta^{1 x}\right)-u_{x t} D_{x}\left(\xi^{1}\right)-u_{x x} D_{t}\left(\xi^{2}\right), \\
\eta^{2 x x} & =D_{x}\left(\eta^{1 x}\right)-v_{x t} D_{x}\left(\xi^{1}\right)-v_{x x} D_{t}\left(\xi^{2}\right), \\
\eta^{1 x x x} & =D_{x}\left(\eta^{1 x x}\right)-u_{x x t} D_{x}\left(\xi^{1}\right)-u_{x x x} D_{t}\left(\xi^{2}\right), \\
\eta^{2 x x x} & =D_{x}\left(\eta^{2 x x}\right)-v_{x x t} D_{x}\left(\xi^{1}\right)-v_{x x x} D_{t}\left(\xi^{2}\right)
\end{aligned}
$$

and the following vector can be used to derive the associated Lie algebra,

$$
\begin{aligned}
V & =\xi^{1}(x, t, u, v) \frac{\partial}{\partial x}+\xi^{2}(x, t, u, v) \frac{\partial}{\partial t} \\
& +\eta^{1}(x, t, u, v) \frac{\partial}{\partial u}+\eta^{2}(x, t, u, v) \frac{\partial}{\partial v} .
\end{aligned}
$$

The $\mathrm{V}$ must meet the following Lie point symmetry condition, and we also figure out the coefficient function of the vector field: $\xi^{1}(x, t, u, v), \xi^{2}(x, t, u, v), \eta^{1}(x, t, u, v)$, $\eta^{2}(x, t, u, v)$ via the following condition.

$$
\left.\operatorname{pr}^{(3)} v(\Delta)\right|_{\Delta=0}=0 .
$$

The invariance condition[34] gives

$$
\left.\xi^{2}(x, t, u, v)\right|_{t=0}=0,
$$

the $\eta_{\alpha}^{0}$ is defined by[32, 33].

$$
\begin{aligned}
\eta_{\alpha}^{0} & =\frac{\partial^{\alpha} \eta}{\partial t^{\alpha}}+\left(\eta_{u}-\alpha D_{t}\left(\xi^{2}\right)\right) \frac{\partial^{\alpha} u}{\partial t^{\alpha}}-u \frac{\partial^{\alpha} \eta_{u}}{\partial t^{\alpha}}+\mu \\
& +\sum_{n=1}^{\infty}\left[\left(\begin{array}{l}
\alpha \\
n
\end{array}\right) \frac{\partial^{\alpha} \eta_{u}}{\partial t^{\alpha}}-\left(\begin{array}{c}
\alpha \\
n+1
\end{array}\right) D_{t}^{n+1}\left(\xi^{2}\right)\right] \times D_{t}^{\alpha-n}(u) \\
& -\sum_{n=1}^{\infty}\left(\begin{array}{l}
\alpha \\
n
\end{array}\right) D_{t}^{n}\left(\xi^{1}\right) D_{t}^{\alpha-n}\left(u_{x}\right),
\end{aligned}
$$

where $\mu$ is defined by

$$
\begin{aligned}
\mu & =\sum_{n=2}^{\infty} \sum_{m=2}^{n} \sum_{k=2}^{m} \sum_{r=0}^{k-1}\left(\begin{array}{l}
\alpha \\
n
\end{array}\right)\left(\begin{array}{l}
n \\
m
\end{array}\right)\left(\begin{array}{l}
k \\
r
\end{array}\right) \frac{1}{k !} \\
& \cdot \frac{t^{n-\alpha}}{\Gamma(n+1-\alpha)} \times[-u]^{r} \frac{\partial^{m}}{\partial t^{m}}\left[u^{k-r}\right] \frac{\partial^{n-m+k}}{\partial t^{n-m} \partial u^{k}} .
\end{aligned}
$$

Next, we apply the above knowledge to analyze the Eq. (1), and the Lie symmetry and optimal system of the Eq. (1) are received in the next chapter.

\section{Lie Symmetry and optimal system}

We make full use of the above Lie symmetry analysis method to research the Eq. (1). Firstly, taking (6) into (1), we have that

$$
\frac{\partial^{\alpha} u^{\star}}{\partial t^{\star} \alpha}-u_{x^{\star} \chi^{\star} x^{\star}}^{\star}-u^{\star} v^{\star} u_{x^{\star}}^{\star}=0,
$$




$$
\frac{\partial^{\alpha} v^{\star}}{\partial t^{\star \alpha}}-v_{x^{*} X^{*} X^{*}}^{\star}-u^{\star} v^{\star} v_{X^{*}}^{\star}=0,
$$

substituting the third prolongation $p r^{(3)}$ that we have previously obtained into the Eq. (1), we get the blow result

$$
\begin{aligned}
& \eta^{1 \alpha, t}-u v \eta^{1 x}-v u_{x} \eta^{1}-u u_{x} \eta^{2}-\eta^{1 x x x}=0, \\
& \eta^{2 \alpha, t}-u v \eta^{2 x}-v v_{x} \eta^{1}-u v_{x} \eta^{2}-\eta^{2 x x x}=0,
\end{aligned}
$$

considering the condition that variables $u_{t}, u_{x}, u_{x x}, u_{x t}, v_{t}$, $v_{x}, v_{x x}, v_{x t}, \ldots$ and $D_{t}^{\alpha-n} u, D_{t}^{\alpha-n} u_{x}, D_{t}^{\alpha-n} v, D_{t}^{\alpha-n} v_{x}$ for $n=1,2, \ldots$ of $u$ are independent, substituting (7) and (11) into consideration, let each power of the derivative of $u, v$ become 0 , we have

$$
\begin{aligned}
& \xi_{u}^{1}=\xi_{t}^{1}=\xi_{u}^{2}=\xi_{x}^{2}=\eta_{u u}^{1}=\eta_{v v}^{2}=0, \\
& \frac{\partial^{\alpha} \eta^{1}}{\partial t^{\alpha}}-u \frac{\partial^{\alpha} \eta_{u}^{1}}{\partial t^{\alpha}}-u v \eta_{x}^{1}-\eta_{x x x}^{1}=0, \\
& \frac{\partial^{\alpha} \eta^{2}}{\partial t^{\alpha}}-v \frac{\partial^{\alpha} \eta_{v}^{2}}{\partial t^{\alpha}}-u v \eta_{x}^{2}-\eta_{x x x}^{2}=0, \\
& -\left(\eta_{u}^{1}-\xi_{x}^{1}\right) u v-v \eta^{1}-u \eta^{2}-\left(3 \eta_{u x x}^{1}-\xi_{x x x}^{1}\right)=0, \\
& -\left(\eta_{v}^{2}-\xi_{x}^{1}\right) u v-v \eta^{1}-u \eta^{2}-\left(3 \eta_{v x x}^{2}-\xi_{x x x}^{1}\right)=0, \\
& 3\left(\eta_{x u}^{1}-\xi_{x x}^{1}\right)=0, \\
& 3\left(\eta_{x v}^{2}-\xi_{x x}^{1}\right)=0, \\
& \alpha \xi_{t}^{2}-3 \xi_{x}^{1}=0, \\
& \left(\begin{array}{l}
\alpha \\
n
\end{array}\right) \frac{\partial^{\alpha} \eta_{u}^{1}}{\partial t^{\alpha}}-\left(\begin{array}{c}
\alpha \\
n+1
\end{array}\right) D_{t}^{n+1}\left(\xi^{2}\right)=0, \\
& \left(\begin{array}{l}
\alpha \\
n
\end{array}\right) \frac{\partial^{\alpha} \eta_{v}^{2}}{\partial t^{\alpha}}-\left(\begin{array}{c}
\alpha \\
n+1
\end{array}\right) D_{t}^{n+1}\left(\xi^{2}\right)=0 .
\end{aligned}
$$

\section{$V_{s} V_{t}-V_{t} V_{s}$, we get}

Table 1) based on the commutator operators $\left[V_{s}, V_{t}\right]=$

The second step is to get the adjoint representations of the vector fields via using the commutator relations in Table 1 and the Lie series

$$
\begin{aligned}
\operatorname{Ad}\left(\exp \left(\epsilon V_{i}\right)\right) V_{j} & =V_{j}-\epsilon\left[V_{i}, V_{j}\right] \\
& +\frac{1}{2} \epsilon^{2}\left[V_{i},\left[V_{i}, V_{j}\right]\right] \ldots,
\end{aligned}
$$

we obtain the adjoint representations of the vector fields (see Table 2).

Table 2: Adjoint representation

\begin{tabular}{ccccc}
\hline $\operatorname{Ad}(\epsilon)$ & $V_{1}$ & $V_{2}$ & $V_{3}$ & $V_{4}$ \\
\hline$V_{1}$ & $V_{1}$ & $V_{2}$ & $V_{3}$ & $V_{4}-\varepsilon V_{1}$ \\
$V_{2}$ & $V_{1}$ & $V_{2}$ & $V_{3}$ & $V_{4}-\frac{3 \varepsilon}{\alpha} V_{2}$ \\
$V_{3}$ & $V_{1}$ & $V_{2}$ & $V_{3}$ & $V_{4}$ \\
$V_{4}$ & $e^{\varepsilon} V_{1}$ & $e^{\frac{3 \varepsilon}{\alpha}} V_{2}$ & $V_{3}$ & $V_{4}$ \\
\hline
\end{tabular}

The final step is to get the optimal system for the Eq. (1)

Solving these equations, we get:

$$
\begin{aligned}
\xi^{1} & =c_{1} x+c_{2}, \\
\xi^{2} & =\frac{3 c_{1}}{\alpha} t+c_{3}, \\
\eta^{1} & =6 c_{4} u, \\
\eta^{2} & =-2 c_{1} v-2 c_{4} v,
\end{aligned}
$$

where $c_{1}, c_{2}, c_{3}, c_{4}$ are arbitrary constants. So, four correlative vector fields are acquired from Eq. (16)

$$
\begin{aligned}
& V_{1}=\frac{\partial}{\partial x}, \\
& V_{2}=\frac{\partial}{\partial t}, \\
& V_{3}=u \frac{\partial}{\partial u}-2 v \frac{\partial}{\partial v}, \\
& V_{3}=x \frac{\partial}{\partial x}+\frac{3 t}{\alpha} \frac{\partial}{\partial t}-2 v \frac{\partial}{\partial v} .
\end{aligned}
$$

Next, we can acquire the optimal system of the Eq. (1) via the method that has been clearly described in Refs [35]. The first step is to get the following commutator table(see from the adjoint representations of the vector fields and the result is as follows

$$
\left\{V_{1}, V_{2}, V_{4}, V_{2} \pm V_{1}, V_{3} \pm V_{1}, V_{3} \pm V_{2}, V_{4} \pm V_{3}\right\}
$$

\section{Similarity reductions}

By simple computation, the following equation

$$
\frac{d x}{x}=\frac{\alpha d t}{3 t}=\frac{d v}{-2 v}
$$

show the similarity variables for the infinitesimal generator $V_{4}$ given by

$$
\begin{aligned}
& u=f(\xi), \\
& v=t^{-\frac{2 \alpha}{3}} g(\xi), \\
& \xi=x t^{-\frac{\alpha}{3}} .
\end{aligned}
$$

Summarize the above discussed in detail, Eq. (1) can be converted to a nonlinear ordinary differential equation. 
We lead into the blow Erdély-Kober fractional (EK) differential operator [22] with the intention of achieving this goal

$$
\left(P_{\sigma}^{\xi^{2}, \alpha} f\right):=\prod_{j=0}^{n-1}\left(\xi^{2}+j-\frac{1}{\sigma} \frac{d}{d \xi}\right)\left(K_{\sigma}^{\xi^{2}+\alpha, n-\alpha} f\right)(\xi),
$$

where

$$
n= \begin{cases}{[\alpha]+1,} & \alpha \nsubseteq N, \\ \alpha, & \alpha \in N,\end{cases}
$$

and the EK fractional integral operator is defined by

$$
\left(K_{\sigma}^{\xi^{2}, \alpha} f\right):=\left\{\begin{array}{l}
\frac{1}{\Gamma(\alpha)} \int_{1}^{\infty}(u-1)^{\alpha-1} u^{-\left(\xi^{2}+\alpha\right)} f\left(\xi u^{\frac{1}{\sigma}}\right) d u, \\
f(\xi),
\end{array}\right.
$$

let $n-1<\alpha<n, n=1,2,3,4, \ldots$ According to the definition of the RL fractional derivative, we get

$$
D_{t}^{\alpha} u=\frac{\partial^{n}}{\partial t^{n}}\left[\frac{1}{\Gamma(n-\alpha)} \int_{1}^{t}(t-s)^{n-\alpha-1} f\left(x s^{-\frac{\alpha}{3}}\right) d s\right],
$$

setting $v=\frac{t}{s}$, then $d s=-\frac{t}{v^{2}} d v$, substituting it into the above equation, we get

$$
\begin{aligned}
D_{t}^{\alpha} u & =\frac{\partial^{n}}{\partial t^{n}} \\
& \cdot\left[\frac{1}{\Gamma(n-\alpha)} \int_{1}^{\infty}(v-1)^{n-\alpha-1} t^{n-\alpha} v^{\alpha-n-1} f\left(\xi v^{\frac{\alpha}{3}}\right) d v\right],
\end{aligned}
$$

according to Eq. (25), we have

$$
D_{t}^{\alpha} u=\frac{\partial^{n}}{\partial t^{n}}\left[t^{n-\alpha}\left(K_{\frac{3}{\alpha}}^{1, n-\alpha} f\right)(\xi)\right] .
$$

Continue to simplify the above equation, consider $\xi=$ $x t^{-\frac{\alpha}{3}}, \varphi \in(0, \infty)$, we acquire

$$
t \frac{\partial}{\partial t} \varphi(\xi)=t x\left(-\frac{\alpha}{3}\right) t^{-\frac{\alpha}{3}-1} \varphi^{\prime}(\xi)=-\frac{\alpha}{3} \xi \frac{\partial}{\partial \xi} \varphi(\xi),
$$

so,

$$
\begin{aligned}
& \frac{\partial^{n}}{\partial t^{n}}\left[t^{n-\alpha}\left(K_{\frac{3}{\alpha}}^{1, n-\alpha} f\right)(\xi)\right] \\
& =\frac{\partial^{n-1}}{\partial t^{n-1}}\left[t^{n-\alpha-1}\left(n-\alpha-\frac{\alpha}{3} \xi \frac{\partial}{\partial \xi}\right)\left(K_{\frac{3}{\alpha}}^{1, n-\alpha} f\right)(\xi)\right] \\
& =\frac{\partial^{n-2}}{\partial t^{n-2}}\left[t^{n-\alpha-2}\left(n-\alpha-\frac{\alpha}{3} \xi \frac{\partial}{\partial \xi}\right)\left(n-\alpha-1-\frac{\alpha}{3} \xi \frac{\partial}{\partial \xi}\right)\right. \\
& \left.\cdot\left(K_{\frac{3}{\alpha}}^{1, n-\alpha} f\right)(\xi)\right] \\
& =\frac{\partial^{n-3}}{\partial t^{n-3}}\left[t^{n-\alpha-3}\left(n-\alpha-\frac{\alpha}{3} \xi \frac{\partial}{\partial \xi}\right)\left(n-\alpha-1-\frac{\alpha}{3} \xi \frac{\partial}{\partial \xi}\right)\right.
\end{aligned}
$$

$$
\begin{aligned}
& \left.\cdot\left(n-\alpha-2-\frac{\alpha}{3} \xi \frac{\partial}{\partial \xi}\right) \times\left(K_{\frac{3}{\alpha}}^{1, n-\alpha} f\right)(\xi)\right] \\
& =\ldots \\
& =t^{-\alpha} \prod_{j=0}^{n-1}\left(1-\alpha+j-\frac{\alpha}{3} \xi \frac{\partial}{\partial \xi}\right)\left(K_{\frac{3}{\alpha}}^{1, n-\alpha} f\right)(\xi) .
\end{aligned}
$$

Substituting EK fractional differential operator Eq. (21) in Eq. (28), we have

$$
\begin{aligned}
& t^{-\alpha} \prod_{j=0}^{n-1}\left(1-\alpha+j-\frac{\alpha}{3} \xi \frac{\partial}{\partial \xi}\right)\left(K_{\frac{\alpha}{\alpha}}^{1, n-\alpha} f\right)(\xi) \\
& =t^{-\alpha}\left(P_{\frac{3}{\alpha}}^{1-\alpha, \alpha} f\right)(\xi) .
\end{aligned}
$$

And by the same logic, we can get:

$$
\begin{aligned}
D_{t}^{\alpha} v & =t^{-\frac{5 \alpha}{3}} \prod_{j=0}^{n-1}\left(1-\frac{5 \alpha}{3}+j\right. \\
& \left.-\frac{\alpha}{3} \xi \frac{\partial}{\partial \xi}\right)\left(K_{\frac{3}{\alpha}}^{1-\frac{2 \alpha}{3}, n-\alpha} g\right)(\xi)=t^{-\frac{5 \alpha}{3}}\left(P_{\frac{3}{\alpha}}^{1-\frac{5 \alpha}{3}, \alpha} g\right)(\xi) .
\end{aligned}
$$

So, Eq. (1) can be converted to the nonlinear ordinary differential equation of fractional order, we obtain

$$
\begin{array}{r}
\left(P_{\frac{3}{\alpha}}^{1-\alpha, \alpha} f\right)(\xi)-f^{\prime \prime \prime}(\xi)-f^{\prime}(\xi) f(\xi) g(\xi)=0, \\
\left(P_{\frac{3}{\alpha}}^{1-\frac{5 \alpha}{3}, \alpha} g\right)(\xi)-g^{\prime \prime \prime}(\xi)-g^{\prime}(\xi) g(\xi) f(\xi)=0 .
\end{array}
$$

\section{Explicit analytical power series solutions}

In the section, the exact explicit solution of the Eq. (31) will be obtained via using the power series method [36, 37]. Power series method is a method for solving ordinary differential equations, especially when the solution of differential equation cannot use elementary function or or its integral expression, we seek other solution, especially power series solution is an approximate solution of the commonly used. Using the power series solution and generalized power series solution can solve many important differential equation in mathematical physics, Set

$$
f(\xi)=\sum_{n=0}^{\infty} a_{n} \xi^{n}, \quad g(\xi)=\sum_{n=0}^{\infty} b_{n} \xi^{n},
$$

we get

$$
\begin{aligned}
f^{\prime}(\xi) & =\sum_{n=0}^{\infty}(n+1) a_{n+1} \xi^{n}, \\
f^{\prime \prime}(\xi) & =\sum_{n=0}^{\infty}(n+1)(n+2) a_{n+2} \xi^{n},
\end{aligned}
$$




$$
\begin{aligned}
f^{\prime \prime \prime}(\xi) & =\sum_{n=0}^{\infty}(n+1)(n+2)(n+3) a_{n+3} \xi^{n}, \\
g^{\prime}(\xi) & =\sum_{n=0}^{\infty}(n+1) b_{n+1} \xi^{n}, \\
g^{\prime \prime}(\xi) & =\sum_{n=0}^{\infty}(n+1)(n+2) b_{n+2} \xi^{n}, \\
g^{\prime \prime \prime}(\xi) & =\sum_{n=0}^{\infty}(n+1)(n+2)(n+3) b_{n+3} \xi^{n} .
\end{aligned}
$$

Substituting Eq. (32) and Eq. (33) into Eq. (31), we get

$$
\begin{aligned}
& \sum_{n=0}^{\infty} \frac{\Gamma\left(2-\alpha+\frac{n \alpha}{3}\right)}{\Gamma\left(2+\frac{n \alpha}{3}\right)} a_{n} \xi^{n} \\
& -\sum_{n=0}^{\infty}(n+1)(n+2)(n+3) a_{n+3} \xi^{n} \\
& -\sum_{n=0}^{\infty} \sum_{k=0}^{n} \sum_{i=0}^{k}(n+1-k) a_{n+1-k} a_{i} b_{k-i} \xi^{n}, \\
& \sum_{n=0}^{\infty} \frac{\Gamma\left(2-\frac{5 \alpha}{3}+\frac{n \alpha}{3}\right)}{\Gamma\left(2-\frac{2 \alpha}{3}\right)} b_{n} \xi^{n}-\sum_{n=0}^{\infty}(n+1)(n+2)(n+3) b_{n+3} \xi^{n} \\
& -\sum_{n=0}^{\infty} \sum_{k=0}^{n} \sum_{i=0}^{k}(n+1-k) b_{n+1-k} a_{i} b_{k-i} \xi^{n},
\end{aligned}
$$

Comparing coefficients in Eq. (34), when $n=0$, we have

$$
\begin{aligned}
& a_{3}=\frac{1}{6}\left[\frac{\Gamma(2-\alpha)}{\Gamma(2)} a_{0}-a_{1} a_{0}^{2}\right], \\
& b_{3}=\frac{1}{6}\left[\frac{\Gamma\left(2-\frac{5 \alpha}{3}\right)}{\Gamma\left(2-\frac{2 \alpha}{3}\right)} b_{0}-b_{1} a_{0} b_{0}\right]
\end{aligned}
$$

when $n \geq 1$, we get

$$
\begin{aligned}
a_{n+3} & =\frac{1}{(n+1)(n+2)(n+3)}\left[\frac{\Gamma\left(2-\alpha+\frac{n \alpha}{3}\right)}{\Gamma\left(2+\frac{n \alpha}{3}\right)} a_{n}\right. \\
& \left.-\sum_{k=0}^{n} \sum_{i=0}^{k}(n+1-k) a_{n+1-k} a_{i} b_{k-i}\right], \\
b_{n+3} & =\frac{1}{(n+1)(n+2)(n+3)}\left[\frac{\Gamma\left(2-\frac{5 \alpha}{3}+\frac{n \alpha}{3}\right)}{\Gamma\left(2-\frac{2 \alpha}{3}+\frac{n \alpha}{3}\right)} b_{n}\right. \\
& \left.-\sum_{k=0}^{n} \sum_{i=0}^{k}(n+1-k) b_{n+1-k} a_{i} b_{k-i}\right],
\end{aligned}
$$

substituting (35), (36) and (37) into (32), we can get the solution in the form of power series for Eq. (32),

$$
\begin{aligned}
f(\xi) & =a_{0}+a_{1} \xi+a_{2} \xi^{2}+a_{3} \xi^{3}+\sum_{n=1}^{\infty} a_{n+3} \xi^{n+3} \\
& =a_{0}+a_{1} \xi+a_{2} \xi^{2}+\frac{1}{6}\left[\frac{\Gamma(2-\alpha)}{\Gamma(2)} a_{0}-a_{1} a_{0}^{2}\right] \xi^{3}
\end{aligned}
$$

$$
\begin{aligned}
& +\sum_{n=1}^{\infty} \frac{1}{(n+1)(n+2)(n+3)}\left[\frac{\Gamma\left(2-\alpha+\frac{n \alpha}{3}\right)}{\Gamma\left(2+\frac{n \alpha}{3}\right)} a_{n}\right. \\
& \left.-\sum_{k=0}^{n} \sum_{i=0}^{k}(n+1-k) a_{n+1-k} a_{i} b_{k-i}\right] \xi^{n+3} \\
g(\xi)= & b_{0}+b_{1} \xi+b_{2} \xi^{2}+b_{3} \xi^{3}+\sum_{n=1}^{\infty} b_{n+3} \xi^{n+3} \\
= & a_{0}+a_{1} \xi+a_{2} \xi^{2}+\frac{1}{6}\left[\frac{\Gamma\left(2-\frac{5 \alpha}{3}\right)}{\Gamma\left(2-\frac{2 \alpha}{3}\right)} b_{0}-b_{1} a_{0} b_{0}\right] \xi^{3} \\
+ & \sum_{n=1}^{\infty} \frac{1}{(n+1)(n+2)(n+3)}\left[\frac{\Gamma\left(2-\frac{5 \alpha}{3}+\frac{n \alpha}{3}\right)}{\Gamma\left(2-\frac{2 \alpha}{3}+\frac{n \alpha}{3}\right)} b_{n}\right. \\
& \left.-\sum_{k=0}^{n} \sum_{i=0}^{k}(n+1-k) b_{n+1-k} a_{i} b_{k-i}\right] \xi^{n+3}
\end{aligned}
$$

where $a_{1}, a_{2}, a_{3}, c_{1}, c_{2}, c_{3}$ is constants.

Finally, the exact explicit solution for Eq. (1) is acquired as below

$$
\begin{aligned}
u(x, t) & =a_{0}+a_{1} x t^{-\frac{\alpha}{3}}+a_{2} x^{2} t^{-\frac{2 \alpha}{3}}+a_{3} x^{3} t^{-\alpha} \\
& +\sum_{n=1}^{\infty} a_{n+3} x^{n+3} t^{-\frac{\alpha(n+3)}{3}} \\
& =a_{0}+a_{1} x t^{-\frac{\alpha}{3}}+a_{2} x^{2} t^{-\frac{2 \alpha}{3}} \\
& +\frac{1}{6}\left[\frac{\Gamma(2-\alpha)}{\Gamma(2)} a_{0}-a_{1} a_{0}^{2}\right] x^{3} t^{-\alpha} \\
& +\sum_{n=1}^{\infty} \frac{1}{(n+1)(n+2)(n+3)}\left[\frac{\Gamma\left(2-\alpha+\frac{n \alpha}{3}\right)}{\Gamma\left(2+\frac{n \alpha}{3}\right)} a_{n}\right. \\
& \left.-\sum_{k=0}^{n} \sum_{i=0}^{k}(n+1-k) a_{n+1-k} a_{i} b_{k-i}\right] x^{n+3} t^{-\frac{-\alpha(n+3)}{3}} \\
v(x, t) & =b_{0} t^{-\frac{2 \alpha}{3}}+b_{1} x t^{-\alpha}+b_{2} x^{2} t^{-\frac{4 \alpha}{3}}+b_{3} x^{3} t^{-\frac{5 \alpha}{3}} \\
& +\sum_{n=1}^{\infty} b_{n+3} x^{n+3} t^{-\frac{-\alpha(n+5)}{3}} \\
& =b_{0} t^{-\frac{2 \alpha}{3}}+b_{1} x t^{-\alpha}+b_{2} x^{2} t^{-\frac{4 \alpha}{3}} \\
& +\frac{1}{6}\left[\frac{\Gamma\left(2-\frac{5 \alpha}{3}\right)}{\Gamma\left(2-\frac{2 \alpha}{3}\right)} b_{0}-b_{1} a_{0} b_{0}\right] x^{3} t^{-\frac{5 \alpha}{3}} \\
& +\sum_{n=1}^{\infty} \frac{\Gamma\left(2-\frac{5 \alpha}{3}+\frac{n \alpha}{3}\right)}{(n+1)(n+2)(n+3)} b_{n} \sum_{i=0} \frac{k\left(2-\frac{2 \alpha}{3}+\frac{n \alpha}{3}\right)}{\left.(n+1-k) b_{n+1-k} a_{i} b_{k-i}\right] x^{n+3} t^{\frac{-\alpha(n+5)}{3}}} .
\end{aligned}
$$




\section{Analysis of the convergence}

Here, the convergence of the PS solution equation (41) for eq. (1) will be investigated. Consider eq. (36) and (37), such that

$$
\begin{aligned}
& \left|a_{n+3}\right| \leq\left(\left|\frac{\Gamma\left(2-\alpha+\frac{n \alpha}{3}\right)}{\Gamma\left(2+\frac{n \alpha}{3}\right)}\right|\left|a_{n}\right|\right. \\
& \left.-\sum_{k=0}^{n} \sum_{i=0}^{k}|(n+1-k)|\left|a_{n+1-k}\right|\left|a_{i}\right|\left|b_{k-i}\right|\right), \\
& \left|b_{n+3}\right| \leq\left(\left|\frac{\Gamma\left(2-\frac{5 \alpha}{3}+\frac{n \alpha}{3}\right)}{\Gamma\left(2-\frac{2 \alpha}{3}+\frac{n \alpha}{3}\right)}\right|\left|b_{n}\right|\right. \\
& \left.-\sum_{k=0}^{n} \sum_{i=0}^{k}|(n+1-k)|\left|b_{n+1-k}\right|\left|a_{i}\right|\left|b_{k-i}\right|\right) .
\end{aligned}
$$

Let us take into consideration an implicit functional system with regard to $\xi$ as follows:

$$
\begin{aligned}
& F(\xi, C)=C-c_{0}-c_{1} \xi-c_{2} \xi^{2}-c_{3} \xi^{3}-M \xi^{3}\left(C-C^{\prime} C B\right), \\
& G(\xi, B)=B-d_{0}-d_{1} \xi-d_{2} \xi^{2}-d_{3} \xi^{3}-N \xi^{3}\left(B-B^{\prime} B C\right) .
\end{aligned}
$$

since $F$ and $G$ are analytic in a neighborhood of $\left(0, c_{0}\right)$ and $\left(0, d_{0}\right)$, where $F\left(0, d_{0}\right)=0, G\left(0, c_{0}\right)=0$ and $\frac{\partial}{\partial C} F\left(0, C_{0}\right) \neq$ $0, \frac{\partial}{\partial B} G\left(0, d_{0}\right) \neq 0$. Then, by the implicit function theorem [40], the convergence is given.

$$
\begin{aligned}
\left|a_{n+3}\right| & \leq M\left(\left|a_{n}\right|\right. \\
& \left.-\sum_{k=0}^{n} \sum_{i=0}^{k}|(n+1-k)|\left|a_{n+1-k}\right|\left|a_{i}\right|\left|b_{k-i}\right|\right), \\
\left|b_{n+3}\right| & \leq N\left(\left|b_{n}\right|-\sum_{k=0}^{n} \sum_{i=0}^{k}|(n+1-k)|\left|b_{n+1-k}\right|\left|a_{i}\right|\left|b_{k-i}\right|\right) .
\end{aligned}
$$

.

where $M, N=\max \left\{e_{1}, e_{2}, e_{3}\right\}$, where $e_{1}, e_{2}, e_{3}$ are arbitrary constants. Take into consideration another PS given as

$$
\begin{aligned}
& C(\xi)=\sum_{n=0}^{\infty} c_{n} \xi^{n}, \\
& B(\xi)=\sum_{n=0}^{\infty} d_{n} \xi^{n}
\end{aligned}
$$

and let $c_{i}=\left|a_{i}\right|, d_{i}=\left|b_{i}\right|, i=0,1,2, \ldots$ Then, we can have

$$
\begin{aligned}
& \left|c_{n+3}\right|=M\left(c_{n}-\sum_{k=0}^{n} \sum_{i=0}^{k}(n+1-k) c_{n+1-k} c_{i} d_{k-i}\right), \\
& \left|d_{n+3}\right|=N\left(d_{n}-\sum_{k=0}^{n} \sum_{i=0}^{k}(n+1-k) d_{n+1-k} c_{i} d_{k-i}\right) .
\end{aligned}
$$

Therefore, it is easily seen that $\left|a_{n}\right| \leq c_{n},\left|b_{n}\right| \leq d_{n}, n=$ $0,1, \ldots$. Furthermore, the series $C(\xi)=\sum_{n=0}^{\infty} c_{n} \xi^{n}$ and $B(\xi)=\sum_{n=0}^{\infty} d_{n} \xi^{n}$ are majorant series of eq. (32). We therefore confirm that the series $C(\xi)$ and $B(\xi)$ have a positive radius of convergence. By some calculations, we have

$$
C(\xi)=c_{0}+c_{1} \xi+c_{2} \xi^{2}+c_{3} \xi^{3}+M\left(\sum_{n=0}^{\infty} c_{n}\right.
$$

$$
\begin{aligned}
& \left.-\sum_{n=0}^{\infty} \sum_{k=0}^{n} \sum_{i=0}^{k}(n+1-k) c_{n+1-k} c_{i} d_{k-i}\right) \xi^{n+3}, \\
B(\xi)= & d_{0}+d_{1} \xi+d_{2} \xi^{2}+d_{3} \xi^{3}+M\left(\sum_{n=0}^{\infty} d_{n}\right. \\
& \left.-\sum_{n=0}^{\infty} \sum_{k=0}^{n} \sum_{i=0}^{k}(n+1-k) d_{n+1-k} c_{i} d_{k-i}\right) \xi^{n+3} .
\end{aligned}
$$

\section{Conservation laws}

In this part, we solve the adjoint equation and Cls by using the related formula, most of the specific knowledge about $\mathrm{Cls}$ has been presented in [37-39]. The form of the Lagrangian is as blow

$$
L=p(x, t)\left(D_{t}^{\alpha} u-u_{x x x}-u v u_{x}\right)+q\left(D_{t}^{\alpha} v-v_{x x x}-u v v_{x}\right) .
$$

In the above equation $p(x, t), q(x, t)$ are another dependent variable. The Euler-Lagrange operator [39] are

$$
\begin{aligned}
& \frac{\delta}{\delta u}=\frac{\partial}{\partial u}+\left(D_{t}^{\alpha}\right)^{\star} \frac{\partial}{\partial D_{t}^{\alpha} u}-D_{x} \frac{\partial}{\partial u_{x}}-D_{x x x} \frac{\partial}{\partial u_{x x x}}, \\
& \frac{\delta}{\delta v}=\frac{\partial}{\partial v}+\left(D_{t}^{\alpha}\right)^{\star} \frac{\partial}{\partial D_{t}^{\alpha} v}-D_{x} \frac{\partial}{\partial v_{x}}-D_{x x x} \frac{\partial}{\partial v_{x x x}},
\end{aligned}
$$

where $\left(D_{t}^{\alpha}\right)^{\star}$ is the adjoint operator of $\left(D_{t}^{\alpha}\right)$, the adjoint equations are given by

$$
\begin{aligned}
& F_{1}^{\star}=\frac{\delta L}{\delta u}=0, \\
& F_{2}^{\star}=\frac{\delta L}{\delta v}=0,
\end{aligned}
$$

combining the above equations, we get:

$$
\begin{aligned}
& F_{1}^{\star}=\frac{\delta L}{\delta u}=p\left(D_{t}^{\alpha}\right)^{\star}-q v v_{x}+p_{x} u v+p u v_{x}+p_{x x x}=0 . \\
& F_{2}^{\star}=\frac{\delta L}{\delta v}=q\left(D_{t}^{\alpha}\right)^{\star}-p u u_{x}+q u_{x} v+q_{x} u v+q_{x x x}=0
\end{aligned}
$$

the adjoint equations of Eq. (1) can be write as below:

$$
p\left(D_{t}^{\alpha}\right)^{\star}-q v v_{x}+p_{x} u v+p u v_{x}+\left.p_{x x x}\right|_{q=\phi^{1}(x, t, u, v)}
$$


$=\lambda_{1}\left(D_{t}^{\alpha} u-u_{x x x}-u v u_{x}\right)$.

$q\left(D_{t}^{\alpha}\right)^{\star}-p u u_{x}+q u_{x} v+q_{x} u v+\left.q_{x x x}\right|_{p=\phi^{2}(x, t, u, v)}$

$=\lambda_{2}\left(D_{t}^{\alpha} v-v_{x x x}-u v v_{x}\right)$

where

$$
\begin{aligned}
q_{x} & =\phi_{x}^{1}+\phi_{u}^{1} u_{x}+\phi_{v}^{1} v_{x} \\
p_{x} & =\phi_{x}^{2}+\phi_{u}^{2} u_{x}+\phi_{v}^{2} v_{x} \\
q_{x x x} & =\phi_{x x x}^{1}+3 \phi_{u u v}^{1} u_{x}^{2} v_{x}+3 \phi_{u v v}^{1} u_{x} v_{x}^{2}+3 \phi_{u u}^{1} u_{x} u_{x x} \\
& +3 \phi_{u v}^{1} u_{x} v_{x x}+3 \phi_{u v}^{1} u_{x x} v_{x}+3 \phi_{v v}^{1} v_{x} v_{x x}+6 \phi_{x u v}^{1} u_{x} v_{x} \\
& +3 \phi_{x x u}^{1} u_{x}+3 \phi_{x x v}^{1} v_{x}+3 \phi_{x u}^{1} u_{x x}+3 \phi_{x v}^{1} v_{x x}+\phi_{u}^{1} u_{x x x} \\
& +\phi_{v}^{1} v_{x x x}+3 \phi_{x u u}^{1} u_{x}^{2}+3 \phi_{x v v}^{1} v_{x}^{2}+\phi_{u u u}^{1} u_{x}^{3}+\phi_{v v v}^{1} v_{x}^{3} \\
q_{x x x} & =\phi_{x x x}^{2}+3 \phi_{u u v}^{2} u_{x}^{2} v_{x}+3 \phi_{u v v}^{2} u_{x} v_{x}^{2}+3 \phi_{u u}^{2} u_{x} u_{x x} \\
& +3 \phi_{u v}^{2} u_{x} v_{x x}+3 \phi_{u v}^{2} u_{x x} v_{x}+3 \phi_{v v}^{2} v_{x} v_{x x}+6 \phi_{x u v}^{2} u_{x} v_{x} \\
& +3 \phi_{x x u}^{2} u_{x}+3 \phi_{x x v}^{2} v_{x}+3 \phi_{x u}^{2} u_{x x}+3 \phi_{x v}^{2} v_{x x}+\phi_{u}^{2} u_{x x x} \\
& +\phi_{v}^{2} v_{x x x}+3 \phi_{x u u}^{2} u_{x}^{2}+3 \phi_{x v v}^{2} v_{x}^{2}+\phi_{u u u}^{2} u_{x}^{3}+\phi_{v v v}^{2} v_{x}^{3}
\end{aligned}
$$

Next, we consider $x, t$ and $u(x, t), v(x, t)$, we get

$$
\bar{X}+D_{t}\left(\xi^{2}\right) l+D_{x}\left(\xi^{1}\right) l=W_{1} \frac{\delta}{\delta u}+W_{2} \frac{\delta}{\delta v}+D_{t} N^{t}+D_{x} N^{x}
$$

In Eq. (53) $l$ is the identity operator, $\frac{\delta}{\delta u}$ is the EulerLagrangian operator, $N^{x}$ and $N^{t}$ are the Noether operators, $\bar{X}$ is as blow

$$
\begin{aligned}
\bar{X} & =\xi^{2} \frac{\partial}{\partial t}+\xi^{1} \frac{\partial}{\partial x}+\eta^{1} \frac{\partial}{\partial u}+\eta^{2} \frac{\partial}{\partial v}+\eta^{1 \alpha, t} \frac{\partial}{\partial D_{t}^{\alpha} u} \\
& +\eta^{2 \alpha, t} \frac{\partial}{\partial D_{t}^{\alpha} v}+\eta^{1 x} \frac{\partial}{\partial u_{x}}+\eta^{2 x} \frac{\partial}{\partial v_{x}}+\eta^{1 x x x} \frac{\partial}{\partial u_{x x x}} \\
& +\eta^{2 x x x} \frac{\partial}{\partial v_{x x x}},
\end{aligned}
$$

and the $W_{1}, W_{2}$ are defined by

$$
\begin{aligned}
& W_{1}=\eta^{1}-\xi^{2} u_{t}-\xi^{1} u_{x} \\
& W_{2}=\eta^{2}-\xi^{2} v_{t}-\xi^{1} v_{x}
\end{aligned}
$$

To the generator $V_{4}$, the corresponding Lie characteristic function can be represented as

$$
\begin{aligned}
& W_{1}=-\frac{3 t}{\alpha} u_{t}-x u_{x}, \\
& W_{2}=-2 v--\frac{3 t}{\alpha} v_{t}-x v_{x} .
\end{aligned}
$$

The operator $N^{t}$ is defined by [37]

$$
\begin{aligned}
N^{t} & =\xi^{2}+\sum_{k=0}^{n-1}(-1)^{k}{ }_{o} D^{\alpha-1-k}\left(W_{1}\right) D_{t}^{k} \frac{\partial}{\partial_{o} D_{t}^{\alpha} u} \\
& -(-1)^{n} J\left(W_{1}, D_{t}^{n} \frac{\partial}{\partial_{o} D_{t}^{\alpha} u}\right) \\
& +\sum_{k=0}^{n-1}(-1)^{k}{ }_{o} D^{\alpha-1-k}\left(W_{2}\right) D_{t}^{k} \frac{\partial}{\partial_{o} D_{t}^{\alpha} v}
\end{aligned}
$$

$$
-(-1)^{n} J\left(W_{2}, D_{t}^{n} \frac{\partial}{\partial_{o} D_{t}^{\alpha} v}\right)
$$

with $J$ given by

$$
J(f, g)=\frac{1}{\Gamma(n-\alpha)} \int_{0}^{t} \int_{t}^{T} \frac{f\left(\xi^{2}, x\right) g(\mu, x)}{\left(\mu-\xi^{2}\right)^{\alpha+1-n}} d \mu d t .
$$

For Eq. (1), the operator $N^{x}$ is defined by

$$
\begin{aligned}
N^{x} & =\xi^{1}+W_{1} \frac{\delta}{\delta u_{x}}+D_{x}\left(W_{1}\right)\left[\frac{\delta}{\delta u_{x x}}\right] \\
& +D_{x x}\left(W_{1}\right)\left[\frac{\delta}{\delta u_{x x x}}\right]+W_{2} \frac{\delta}{\delta v_{x}} \\
& +D_{x}\left(W_{2}\right)\left[\frac{\delta}{\delta v_{x x}}\right]+D_{x x}\left(W_{2}\right)\left[\frac{\delta}{\delta v_{x x x}}\right] \\
& =\xi^{1}+W_{1}\left[\frac{\partial}{\partial u_{x}}+D_{x x} \frac{\partial}{\partial u_{x x x}}\right] \\
& +D_{x}\left(w_{1}\right)\left[\frac{\partial}{\partial u_{x x}}-D_{x} \frac{\partial}{\partial u_{x x x}}\right]+D_{x x}\left(W_{1}\right)\left[\frac{\partial}{\partial u_{x x x}}\right] \\
& +W_{2}\left[\frac{\partial}{\partial v_{x}}+D_{x x} \frac{\partial}{\partial v_{x x x}}\right]+D_{x}\left(w_{2}\right)\left[\frac{\partial}{\partial v_{x x}}-D_{x} \frac{\partial}{\partial v_{x x x}}\right] \\
& +D_{x x}\left(W_{2}\right)\left[\frac{\partial}{\partial v_{x x x}}\right] .
\end{aligned}
$$

Substituting (1) into (53), we get:

$$
\left.\left(\bar{X} L+D_{t}\left(\xi^{2}\right) L+D_{X}\left(\xi^{1}\right) L\right)\right|_{E q .(1)}=0,
$$

hence, the form of the Cls for Eq. (1) can be written as

$$
D_{t}\left(N^{t} L\right)+D_{x}\left(N^{\chi} L\right)=0 \text {. }
$$

Next, according to the basic definitions present above, we acquire the Cls for Eq. (1), and divide into the following cases to discuss:

Case 1. For $\alpha \in(0,1)$, the components of the conserved vector are

$$
\begin{aligned}
C_{i}^{t} & =N^{t} L=\xi^{2} L+(-1)^{0}{ }_{o} D^{\alpha-1}\left(W_{1}\right) D_{t}^{0} \frac{\partial L}{\partial_{o} D_{t}^{\alpha} u}-(-1)^{1} \\
& \times J\left(W_{1}, D_{t}^{1} \frac{\partial L}{\partial_{o} D_{t}^{\alpha} u}\right)+(-1)^{0}{ }_{o} D^{\alpha-1}\left(W_{2}\right) D_{t}^{0} \frac{\partial L}{\partial{ }_{o} D_{t}^{\alpha} v} \\
& -(-1)^{1} \times J\left(W_{2}, D_{t}^{1} \frac{\partial L}{\partial{ }_{o} D_{t}^{\alpha} v}\right)={ }_{o} D^{\alpha-1}\left(W_{1}\right) p \\
& +J\left(W_{1}, p_{t}\right)+{ }_{o} D^{\alpha-1}\left(W_{2}\right) q+J\left(W_{2}, q_{t}\right), \\
C_{i}^{x} & =N^{x} L=\xi^{1} L+W_{1}\left[\frac{\partial L}{\partial u_{x}}+D_{x x} \frac{\partial L}{\partial u_{x x x}}\right] \\
& +D_{x}\left(w_{1}\right)\left[\frac{\partial L}{\partial u_{x x}}-D_{x} \frac{\partial L}{\partial u_{x x x}}\right]+D_{x x}\left(W_{1}\right)\left[\frac{\partial L}{\partial u_{x x x}}\right] \\
& +W_{2}\left[\frac{\partial L}{\partial v_{x}}+D_{x x} \frac{\partial L}{\partial v_{x x x}}\right]+D_{x}\left(w_{2}\right)\left[\frac{\partial L}{\partial v_{x x}}-D_{x} \frac{\partial L}{\partial v_{x x x}}\right]
\end{aligned}
$$




$$
\begin{aligned}
& +D_{x x}\left(W_{2}\right)\left[\frac{\partial L}{\partial v_{x x x}}\right]=-W_{1}\left(q u v+q_{x x}\right)+q_{x} D_{x}\left(W_{1}\right) \\
& -q D_{x x}\left(W_{1}\right)-W_{2}\left(p u v+p_{x x}\right)+p_{x} D_{x}\left(W_{2}\right)-p D_{x x}\left(W_{2}\right),
\end{aligned}
$$

where the functions $W_{1}, W_{2}$ are given by

$$
\begin{aligned}
& W_{1}=-\frac{3 t}{\alpha} u_{t}-x u_{x}, \\
& W_{2}=-2 v--\frac{3 t}{\alpha} v_{t}-x v_{x} .
\end{aligned}
$$

Case 2. When $\alpha \in(1,2)$, the components of the conserved vector are

$$
\begin{aligned}
C_{i}^{t} & =N^{t} L=\xi^{2} L+(-1)^{0}{ }_{o} D^{\alpha-1}\left(W_{1}\right) D_{t}^{0} \frac{\partial L}{\partial{ }_{o} D_{t}^{\alpha} u} \\
& +(-1)^{1}{ }_{o} D^{\alpha-2}\left(W_{1}\right) D_{t}^{1} \frac{\partial L}{\partial_{o} D_{t}^{\alpha} u}-(-1)^{1} \\
& \times J\left(W_{1}, D_{t}^{1} \frac{\partial L}{\partial{ }_{o} D_{t}^{\alpha} u}\right)-(-1)^{2} \times J\left(W_{1}, D_{t}^{2} \frac{\partial L}{\partial_{o} D_{t}^{\alpha} u}\right) \\
& +(-1)^{0}{ }_{o} D^{\alpha-1}\left(W_{2}\right) D_{t}^{0} \frac{\partial L}{\partial_{o} D_{t}^{\alpha} v} \\
& +(-1)^{1}{ }_{o} D^{\alpha-2}\left(W_{2}\right) D_{t}^{1} \frac{\partial L}{\partial_{o} D_{t}^{\alpha} v}-(-1)^{1} \\
& \times J\left(W_{2}, D_{t}^{1} \frac{\partial L}{\partial{ }_{o} D_{t}^{\alpha} v}\right)-(-1)^{2} \times J\left(W_{2}, D_{t}^{2} \frac{\partial L}{\partial{ }_{o} D_{t}^{\alpha} v}\right) \\
& ={ }_{o} D^{\alpha-1}\left(W_{1}\right) p-{ }_{o} D^{\alpha-2}\left(W_{1}\right) p_{t}+J\left(W_{1}, p_{t}\right) \\
& -J\left(W_{1}, p_{t t}\right)+{ }_{o} D^{\alpha-1}\left(W_{2}\right) q-{ }_{o} D^{\alpha-2}\left(W_{2}\right) q_{t}+J\left(W_{2}, q_{t}\right) \\
& -J\left(W_{2}, q_{t t}\right), \\
C_{i}^{x} & =N^{x} L=\xi^{1} L+W_{1}\left[\frac{\partial L}{\partial u_{x}}+D_{x x} \frac{\partial L}{\partial u_{x x x}}\right] \\
& +D_{x}\left(w_{1}\right)\left[\frac{\partial L}{\partial u_{x x}}-D_{x} \frac{\partial L}{\partial u_{x x x}}\right]+D_{x x}\left(W_{1}\right)\left[\frac{\partial L}{\partial u_{x x x}}\right] \\
& +W_{2}\left[\frac{\partial L}{\partial v_{x}}+D_{x x} \frac{\partial L}{\partial v_{x x x}}\right]+D_{x}\left(w_{2}\right)\left[\frac{\partial L}{\partial v_{x x}}-D_{x} \frac{\partial L}{\partial v_{x x x}}\right] \\
& +D_{x x}\left(W_{2}\right)\left[\frac{\partial L}{\partial v_{x x x}}\right]=-W_{1}\left(q u v+q_{x x}\right)+q_{x} D_{x}\left(W_{1}\right) \\
& -q D_{x x}\left(W_{1}\right)-W_{2}\left(p u v+p_{x x}\right)+p_{x} D_{x}\left(W_{2}\right)-p D_{x x}\left(W_{2}\right),
\end{aligned}
$$

where the functions $W_{1}, W_{2}$ are given by

$$
\begin{aligned}
& W_{1}=-\frac{3 t}{\alpha} u_{t}-x u_{x}, \\
& W_{2}=-2 v--\frac{3 t}{\alpha} v_{t}-x v_{x} .
\end{aligned}
$$

\section{Concluding remarks}

In the paper, we studied the time fractional equations which were the extension of the mkdv equations. Firstly, the Lie symmetries and optimal systems of the equations are completely presented, and the equations were reduced to the nonlinear ordinary differential equations of fractional order. Then we get explicit solutions of the equations by applying the power series approach. And we study the convergence of the exact solutions for the equations. Finally, we use the symmetries and adjoint equations to construct the conservation laws of the governing equations.

Acknowledgement: Project supported by the Natural Science Foundation of Jiangsu Province under Grant No. BK20170171

\section{References}

[1] Olver P.J., Application of Lie Group to Differential Equation, 1986, Springer, New York.

[2] Grosser M., Kunzinger M., Oberguggenberger M., et al., Applications to Lie Group Analysis of Differential Equations// Geometric Theory of Generalized Functions with Applications to General Relativity. Springer Netherlands, 2001:353-414.

[3] Miller W., Ovsiannikov L. V., Group Analysis of Differential Equations. 1982, 92(2):155.

[4] Meinhardt J. R., Symmetries and differential equations, 1980, 28(15):507-514.

[5] Liu H., Li J., Zhang Q., Lie symmetry analysis and exact explicit solutions for general Burgers' equation, Journal of Computational \& Applied Mathematics, 2009, 228(1):1-9.

[6] Liu H., Li J., Lie Symmetry Analysis and Exact Solutions for the Extended mKdV Equation, Acta Applicandae Mathematicae, 2010, 109(2):1107-1119.

[7] Tu J.M., Tian S.F., Xu M.J., et al., On Lie symmetries, optimal systems and explicit solutions to the KudryashovSinelshchikov equation, Applied Mathematics \& Computation, 2016, 275(C):345-352.

[8] Liu H., Geng Y., Symmetry reductions and exact solutions to the systems of carbon nanotubes conveying fluid, Journal of Differential Equations, 2013, 254(5):2289-2303.

[9] Feng L.L., Tian S.F., Zhang T.T., et al., Lie symmetries, conservation laws and analytical solutions for two-component integrable equations, Chinese Journal of Physics, 2017, 55(3).

[10] Nadjafikhah M., ShirvaniSh V., Lie symmetries and conservation laws of the Hirota CRamani equation, Communications in Nonlinear Science \& Numerical Simulation, 2012, 17(11):4064-4073.

[11] Gardner C.S., Greene J.M., Kruskal M.D., et al., Method for Solving the Korteweg-deVries Equation, Phys.rev.lett, 1967, 19(19):1095- 1097.

[12] Estcez P.G., Conde E., Gordoa P.R., Unified approach to Miura, Backlund and Darboux Transformations for Nonlinear Partial Differential Equations. Journal of Nonlinear Mathematical Physics, 1998, 5(5):82.

[13] Wazwaz A.M., Multiple-soliton solutions for the KP equation by Hirota bilinear method and by the tanh C coth method, Applied Mathematics \& Computation, 2007, 190(1):633-640.

[14] Liu H., Li J., Chen F., Exact periodic wave solutions for the hKdV equation, Nonlinear Analysis, 2009, 70(6):2376-2381. 
[15] Sahoo S., Ray S.S., Lie symmetry analysis and exact solutions of $(3+1)$ dimensional Yu Toda Sasa Fukuyama equation in mathematical physics, Pergamon Press, Inc. 2017.

[16] Zhang Y., Lie Symmetry Analysis and Exact Solutions of the Sharma-Tasso-Olever Equation, International Journal of Applied Mathematics, 2016.

[17] Giresunlu I.B., Sag lam Ozkan Y., Yas,ar E., On the exact solutions, lie symmetry analysis, and conservation laws of Schamel- CKortewegCde Vries equation, Mathematical Methods in the Applied Sciences, 2017, 40.

[18] Gazizov R.K., Ibragimov N.H., Lie Symmetry Analysis of Differential Equations in Finance, Nonlinear Dynamics, 1998, 17(4):387407.

[19] Wang G.W., Liu X.Q., Zhang Y.Y., s Lie symmetry analysis to the time fractional generalized fifth-order KdV equation, Communications in Nonlinear Science \& Numerical Simulation, 2013, 18(9):2321-2326.

[20] Janocha D.D., Wactawczyk M., Oberlack M., Lie Symmetry Analysis of the Hopf Functional-Differential Equation, Symmetry, 2015, 7(3):1536-1566.

[21] Clarkson P.A., Kruskal M.D., New similarity reductions of the Boussinesq equation, Journal of Mathematical Physics, 1989, 30(10):2201-2213.

[22] Kiryakova V., Generalized Fractional Calculus and Applications, Pitman Research Notes in Mathmatics, 1994, 301.

[23] Podlubny I., Fractional Differential Equations, Mathematics in Science and Engineering, 1999.

[24] Feng L.L., Tian S.F., Zhang T.T., etal., Lie symmetries, conservation laws and analytical solutions for twocomponent integrable equations, Chinese Journal of Physics, 2017:S0577907317300862.

[25] Wang G.W., Xu T.Z., Feng T., Lie Symmetry Analysis and Explicit Solutions of the Time Fractional Fifth-Order KdV Equation. Plos One, 2014, 9(2):e88336.

[26] Baleanu D., Inc M., Yusuf A., et al., Lie symmetry analysis, exact solutions and conservation laws for the time fractional modified Zakharov C Kuznetsov equation, Nonlinear Analysis Modelling \& Control, 2017, 22(6):861C876.

[27] Qin C.Y., Tian S.F., Wang X.B., et al., Lie symmetry analysis, conservation laws and explicit solutions for the time fractional Rosenau- Haynam equation, Waves in Random Media, 2016, 27(2):308- 324.
[28] Wang X.B., Tian S.F., Qin C.Y., etal., Lie Symmetry Analysis, Analytical Solutions, and Conservation Laws of the Generalised Whitham-CBroerCKaupC Like Equations, Zeitschrift Naturforschung Teil A, 2017, 72(3).

[29] Qin C.Y., Tian S.F., Wang X.B., et al., Lie symmetry analysis, conservation laws and analytic solutions of the time fractional Kolmogorov-Petrovskii-Piskunov equation, Chinese Journal of Physics, 2018.

[30] Baleanu D., Inc M., Yusuf A., et al., Space-time fractional Rosenou- Haynam equation: Lie symmetry analysis, explicit solutions and conservation laws, Advances in Difference Equations, 2018, 2018(1):46.

[31] Chen C., Jiang Y.L., Lie Group Analysis, Exact Solutions and New Conservation Laws for Combined KdVCmKdV Equation, Differential Equations \& Dynamical Systems, 2017:1-14.

[32] Xu M.J., Tian S.F., Tu J.M., et al., Bac̈klund transformation, infinite conservation laws and periodic wave solutions to a generalized (2+1)-dimensional Boussinesq equation, Nonlinear Analysis Real World Applications, 2016, 31:388-408.

[33] Wang X.B., Tian S.F., Qin C.Y., et al., Characteristics of the solitary waves and rogue waves with interaction phenomena in a generalized ( 3+1 3+1 math Container Loading Mathjax )-dimensional Kadomtsev C Petviashvili equation, Applied Mathematics Letters, 2017, 72.

[34] Gazizov R.K., Kasatkin A.A., Lukashchuk S.Y., Symmetry properties of fractional diffusion equations, Physica Scripta, 2009, 2009(T136):014016.

[35] Kudryashov N.A., Sinelshchikov D.I., Equation for three- dimensional nonlinear waves in liquid with gas bubbles, Physica Scripta, 2012, 85(2):25402-25409(8).

[36] Galaktionov V.A., Svirshchevskii S. R., Exact solutions and invariant subspaces of nonlinear partial differential equations in mechan-ics and physics, Chapman \& Hall/CRC, 2007.

[37] Noether E., Invariant variation problems, Transport Theory \& Statistical Physics, 2005, 1(3):186-207.

[38] Singla K., Gupta R. K., On invariant analysis of some time fractional nonlinear systems of partial differential equations, I. Journal of Mathematical Physics, 2016, 57(10):1309-1322.

[39] Ibragimov N.H., A new conservation theorem, Journal of Mathematical Analysis \& Applications, 2007, 333(1):311-328.

[40] Rudin W., Principles of Mathematical Analysis, 3rd ed. (China Machine Press, Beijing, 2004). 